\title{
Outcomes of Stereotactic Body Radiotherapy for Hepatocellular Carcinoma with Severe Cirrhosis and Ineligibility for Transplant
}

\author{
VLADIMIR VALAKH, STEVEN GRESSWELL and ALEXANDER KIRICHENKO \\ Allegheny Health Network, Division of Radiation Oncology, Pittsburgh, PA, U.S.A.
}

\begin{abstract}
Background/Aim: Our study reviewed the results of patients with hepatocellular carcinoma and Child-Pugh score 8-11 cirrhosis treated with stereotactic body radiotherapy when liver transplant was not an option. Patients and Methods: A retrospective review was performed on 15 patients with Child-Pugh class $B$ and $C$ cirrhosis treated with stereotactic body radiotherapy. The median total dose was 35 Gy in 4-5 fractions. None were listed for a liver transplant due to either being outside of the Milan criteria or to medical contraindications. Results: The overall survival was $26.7 \%$ at 6 months, with a mean survival of 152 days. The mean survival with and without ascites was 3.3 months and 8.3 months, respectively. Conclusion: For hepatocellular carcinoma with cirrhosis of Child-Pugh score 8 or more, prognosis after liver stereotactic body radiotherapy was suboptimal. While irradiation achieved local tumor control, progressive cirrhosis was a common cause of death. Patients without ascites at the time of radiotherapy had the best prognosis.
\end{abstract}

Hepatocellular carcinoma (HCC) affects individuals with liver cirrhosis disproportionally more often than those with unaltered liver function $(1,2)$. For patients with simultaneous diagnoses of cirrhosis and liver-confined HCC, orthotopic liver transplantation (OLT) is the definitive treatment of choice (3). The probability of successful hepatic transplantation has been correlated with favorable clinical and radiographic HCC characteristics (4). Therefore, taking into account the limited organ availability, transplantation is restricted to patients with HCC meeting strict criteria relating

Correspondence to: Vladimir Valakh, MD, Allegheny Health Network, Division of Radiation Oncology, 4800 Friendship Avenue, Pittsburgh, PA 15212, USA. Tel: +1 4127991021, Fax: +1 4125781936, e-mail: vladimir.valakh@ahn.org

Key Words: Stereotactic radiotherapy, hepatocellular carcinoma, cirrhosis. to tumor size, number, and involvement of hepatic structures (5). Additionally, a significant proportion of patients with HCC cannot be considered for OLT due to other contraindications for transplant, such as cardiopulmonary comorbidities or ongoing substance abuse.

For patients with co-existing hepatic cirrhosis and nonmetastatic HCC who are not transplant candidates, various tumor-directed therapies are available. They include, but are not limited to transarterial chemo-embolization (TACE), percutaneous ablative therapies, systemic chemotherapy, intraarterial radioembolization, and external beam radiotherapy (6, 7). Select patients with good response to these therapies may be re-considered for a liver transplant (7).

External-beam radiotherapy is an attractive modality of HCC treatment. Advantages of radiation therapy include its non-invasiveness, patient convenience, low cost, and effectiveness (8-13). In the United States, stereotactic body radiotherapy (SBRT) is one of the most popular forms of liver-directed radiotherapy for $\operatorname{HCC}(14,15)$. SBRT entails delivery of 3 to 5 fractions of relatively high doses of radiation to a precisely identified target, simultaneously limiting unwanted exposure of tissues not affected by cancer, such as the benign liver parenchyma.

However, the liver is one of the most radiosensitive organs. Use of radiotherapy, including SBRT, for HCC has therefore been traditionally constrained by the concerns of excessive radiation toxicity (16). In particular, the cirrhotic liver is believed to have lower radiation tolerance compared to the healthy liver. In fact, the currently accruing North American phase III study restricts SBRT use for HCC in patients with cirrhosis to those with Child-Pugh $(\mathrm{CP})$ class A cirrhosis (CP score 5 or 6 only) (17). Limited retrospective data exist on SBRT safety in early Child-Pugh B cirrhosis, usually defined as CP score of 7. The role of SBRT to the liver for patients with severe cirrhosis [Child-Pugh class B (CP score of 8 or 9) and class $\mathrm{C}$ ] has not been yet established.

At our Institution, therefore, SBRT is not routinely recommended for patients with $\mathrm{HCC}$ with underlying severe cirrhosis. Nevertheless, SBRT has been offered for highly 
selected patients with high CP score (CP class B or C) based on an individualized determination by consulting physicians that an acceptable outcome was possible. Some of the factors used in such decision-making process have included good/excellent performance status, HCC location in a single region of the liver, the absence of signs of rapidly progressing hepatic insufficiency (such as worsening hyperbilirubinemia or variceal bleeding), and good social support. This study reviewed our results of SBRT for HCC in the setting of cirrhosis with $\mathrm{CP}$ score of 8 or more.

\section{Patients and Methods}

Fifteen patients with severe cirrhosis (CP score $\geq 8$ ) consecutively received SBRT for de novo, radiographically persistent, or progressive HCC in 2011-2015. Those receiving protracted fractionated radiotherapy instead of SBRT were not included in this study. Six patients with CP score $\geq 8$ who received SBRT as a bridge to transplant during the same time period and ultimately received OLT were also excluded. Clinicopathological characteristics and treatment details were retrospectively retrieved with approval from the Institutional Review Board (approval number RC-5758). None of the patients were lost to follow-up. The patient details are summarized in Table I.

The median age at diagnosis was 62 years. Fourteen were male. All patients were formally evaluated for a liver transplant at an accredited center, but none were listed at the time of radiotherapy due to being outside of the Milan criteria $(\mathrm{n}=10)$ or other transplant contraindications $(n=5)$. Prior to SBRT, six patients received TACE for a median total of three sessions (range=2-4). Five out of six patients treated with TACE experienced a decline in CP score after TACE, with a median decrease of 1 (range $=1-3$ ) CP point between the initiation of TACE and start of radiotherapy. The median time between HCC diagnosis and SBRT was 8 months (range=1-61 months).

At the time of SBRT, 13 patients had CP class B cirrhosis and two class $\mathrm{C}$, with a median $\mathrm{CP}$ score of 8 (range=8-11). The underlying causes of cirrhosis were hepatitis $\mathrm{C}$ infection or alcohol use in all patients. There were no distant metastases from $\mathrm{HCC}$ and one patient had contiguous extrahepatic tumor extension. Three patients had portal vein thrombosis. Seven out of the 15 exhibited clinical symptoms of decompensated cirrhosis within 30 days of initiation of radiotherapy, such as worsening ascites or development of encephalopathy. The median Model for End-Stage Liver Disease (MELD) score at SBRT was 15 (range=9-19), adjusted for serum sodium concentration. None of the patients were receiving hemodialysis or platelet transfusion before SBRT.

SBRT was prescribed to a median total dose of $35 \mathrm{~Gy}$ (range=27.5-50 Gy) in five once-daily fractions $(n=14)$ or four fractions $(n=1)$. The median radiotherapy course duration was 10 calendar days (range $=5-18$ days). Fourteen patients had single radiotherapy targets, with median largest tumor dimension of 3.7 $\mathrm{cm}$ (range=1.9-6.2 cm) and one patient had two separate targets. The median radiotherapy planned target volume was $100.4 \mathrm{~cm}^{3}$.

The particulars of the radiotherapy planning and delivery process have been published elsewhere (18). Briefly, each patient was immobilized with a custom-molded vacuum cushion (Bionix, Toledo, OH, USA) and treatment planning computed tomography (CT) was obtained to define the gross tumor volume (GTV). This was immediately followed by a $4 \mathrm{D}-\mathrm{CT}$ to delineate the internal target volume accounting for respiratory-induced tumor motion. The planning target volume (PTV) was constructed by adding an additional margin to the internal target volume to account for setup error. The SBRT dose was prescribed to the isodose line encompassing the PTV (generally $\geq 90 \%$ ) allowing up to $120 \%$ point dose to the target volume. Image-guided radiation therapy using cone-beam CT was performed before each daily session to reduce set-up uncertainties. Implanted fiducial markers were not used.

Ten out of 15 patients in this study underwent 3D-CT/single photonemission computed tomography (SPECT) to identify anatomic areas of the liver with good residual function. Liver dose constraints were imposed on residual functional liver volume defined on 3D-CT/SPECT with the calculation of predicted functional liver volume ( $p F L V$ ) from an equation used in transplant surgery and ${ }^{90} \mathrm{Y}$ radioembolization dosimetry (pFLV=-794.41+1268.28xbody surface area) $(19,20)$. Next, we specified that at least $35 \%$ of the pFLV from treatment-planning 3D-CT/SPECT should receive no more than 16 Gy for 4 -fraction SBRT or 18 Gy for 5-fraction SBRT. Thirty-five percent of the residual functional liver to be avoided from threshold irradiation corresponds to a conservative estimate of normal liver volume to be spared from hepatic resection (18). Additional constraints included stomach V25 $<10 \mathrm{~cm}^{3}$ (maximum<30 Gy); and small bowel V20 $<20 \mathrm{~cm}^{3}$ (maximum $<30 \mathrm{~Gy}$ ), where V25 and V20 were the corresponding organ volumes receiving at least 25 or $20 \mathrm{~Gy}$, respectively. Adverse reactions were graded according to the Common Terminology Criteria for Adverse Events manual version 4.03.

Sorafenib was given prior to SBRT to one patient, concurrently to one, and sequentially after radiotherapy to another. Two individuals had additional liver-directed radiotherapy during the follow-up period. No other cancer-directed treatments were given.

The duration of survival was calculated from the last day of SBRT, and was estimated by the Kaplan-Meier method. Survival for subgroups was compared using the log-rank test. Calculations were performed using SPSS 20.0 (IBM Corp., Armonk, NY, USA).

\section{Results}

Four out of the 15 patients had acute grade 1 or 2 gastrointestinal side-effects of SBRT but there were no severe acute reactions. One patient did not complete the entire prescribed SBRT course due to death. None of the patients were formally diagnosed with radiation-induced liver disease during the follow-up period. Mild or moderate increase in serum transaminase level after SBRT was noted in four out of the 15 individuals.

All 15 patients had complete follow-up data available for analysis. Death was documented for $13 / 15$ patients in the study. Four patients died within 20 days of the last fraction of SBRT. The immediate cause of death could not be accurately ascertained for the majority of patients. However, the most commonly documented cause of death was hepatic failure. Median follow-up duration for those surviving more than 20 days was 6 months (range=3-13 months). None of the patients experienced radiographic progression of the target HCC after radiotherapy, thus freedom from in-field tumor failure was $100 \%$. Estimated freedom from intrahepatic cancer relapse was $91 \%$ at 6 months. 
Table I. Clinical, laboratory and treatment details for study patients $(N=15)$.

\begin{tabular}{lc}
\hline Characteristic & Median (range) \\
\hline At initial diagnosis of HCC & \\
Age, years & $62(48-77)$ \\
Number of discrete tumors & $2(1-3)$ \\
Largest tumor size, cm & $2.9(1.9-5.5)$ \\
Child-Pugh score & $8(6-10)$ \\
At SBRT initiation & \\
Largest tumor size, cm & $3.7(1.9-6.2)$ \\
Serum total bilirubin level, mg/dI & $1.9(0.7-6.7)$ \\
Serum albumin level, g/dI & $2.7(2.2-3.4)$ \\
Standardized prothrombin time, INR & $1.4(1.1-1.7)$ \\
Child-Pugh score & $8(8-11)$ \\
Serum creatinine level, mg/dl & $1.02(0.62-2.3)$ \\
MELD score & $15.0(9-19.6)$ \\
Total prescribed dose, Gy & $35(27.5-50)$ \\
Planned target volume, $\mathrm{cm}{ }^{3}$ & $100.4(16.8-284)$ \\
\hline
\end{tabular}

HCC: Hepatocellular carcinoma; INR: international normalized ratio; SBRT: stereotactic body radiotherapy; MELD: Model for End-stage Liver Disease.

The overall survival rate for the entire study group at 6 months was $26.7 \%$ (95\% confidence interval $=4.4-49 \%$, Figure 1 ), with mean survival of 152 days. The mean survival for six patients with a history of TACE before SBRT was 6.3 months vs. 4.4 months for those who did not have TACE $(p=0.26$, Figure 2A). The mean survival for patients with CP score of 8 was 5.6 months vs. 3.7 months for those with CP score of 9 or more ( $p=0.6$, Figure $2 \mathrm{~B}$ ). The mean survival for patients with any degree of ascites at the time of SBRT was 3.3 months $v s$. 8.3 months for those without ( $p=0.056$, Figure $3 \mathrm{~A}$ ). For the six patients in our study who had either severe or uncontrolled ascites at the time of the SBRT, the average survival was 66 days. For individuals with any clinical signs of decompensated cirrhosis, the average survival was 3.6 months vs. 6.2 months for those who did not have such symptoms ( $p=0.33$, Figure $3 B)$. No numerically significant effect on survival was noted for MELD score, total serum bilirubin level, the reason for no transplantation, PTV size, total delivered radiation dose or any other factors assessed in this study.

\section{Discussion}

We reported the survival outcomes for 15 patients that were offered SBRT for non-metastatic HCC, as liver transplantation was not possible. The unique unifying feature for this group was pre-existing severe hepatic cirrhosis, namely Child-Pugh class B or C disease with CP score of 8 or more. Traditionally, although high-level published evidence is lacking, this clinical setting would not be considered suitable for liver-directed radiotherapy $(8,9,21)$.

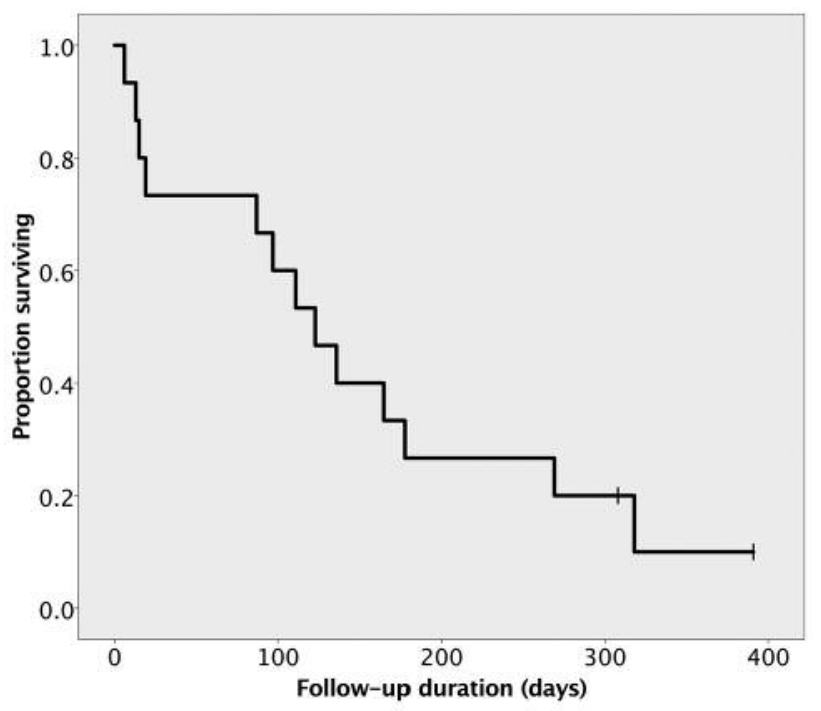

Figure 1. Overall survival for the entire group. Censored patients are indicated by vertical bars.

Indeed, with 20-day post-treatment mortality of $27 \%(4 / 15)$ and mean survival duration of 5 months, the overall outcomes in our study were suboptimal. For patients with competing mortality risks of advanced HCC and severe cirrhosis, a broadly similar prognosis would probably be expected with best supportive care (21). Notably, neither radiation-induced liver disease nor any negative impact on survival according to radiotherapy dose and volume were documented. Thus, it is not likely that radiotherapy hastened the demise of these patients. Conversely, as no patient experienced radiographic progression of the target HCC after radiotherapy, pain associated with growing tumor was prevented.

Our results are complementary to other published case reports of severe cirrhosis that excluded transplant patients. Culleton et al. reported on retrospective outcomes in patients with Child-Pugh B or C HCC treated with SBRT. They noted median survival of 9.9 months for 20 patients with Child-Pugh B (CP score of 7) (21). In contrast, the median survival for nine patients with $\mathrm{CP}$ of 8 or more was only 2.8 months. It was concluded that while a CP score of 7 is suitable for further application of SBRT, SBRT was not recommended at their institution for those with $\mathrm{CP}$ of 8 or more, outside of bridge to transplant. Our study of 15 patients reinforces their observation and allows for further insight into the impact of SBRT on patients with HCC with advanced liver disease.

Although our sample size was small, we attempted exploratory subgroup analyses. One interesting finding was that patients with a history of TACE before radiotherapy had numerically longer survival compared to those who received 
A

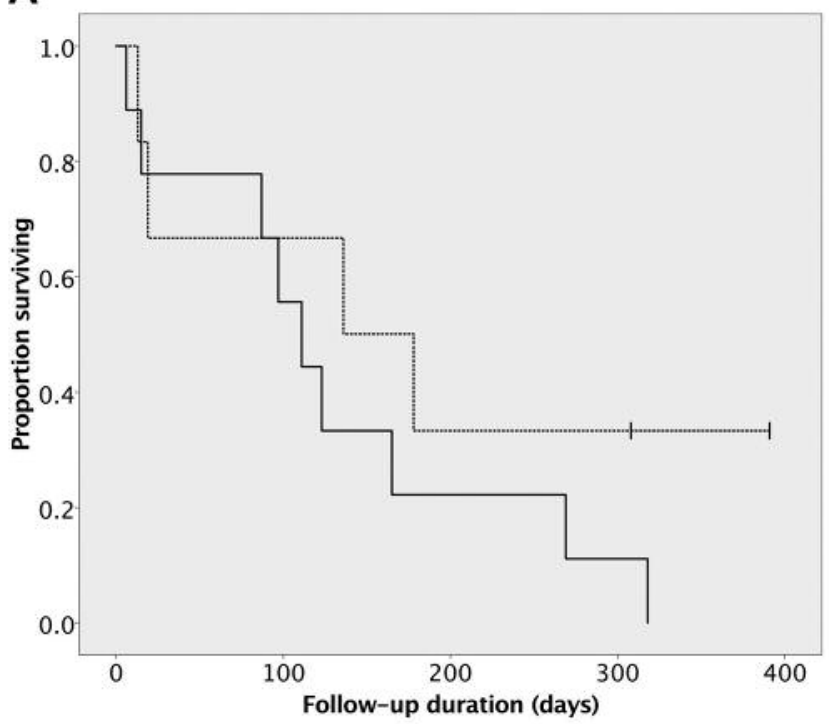

B

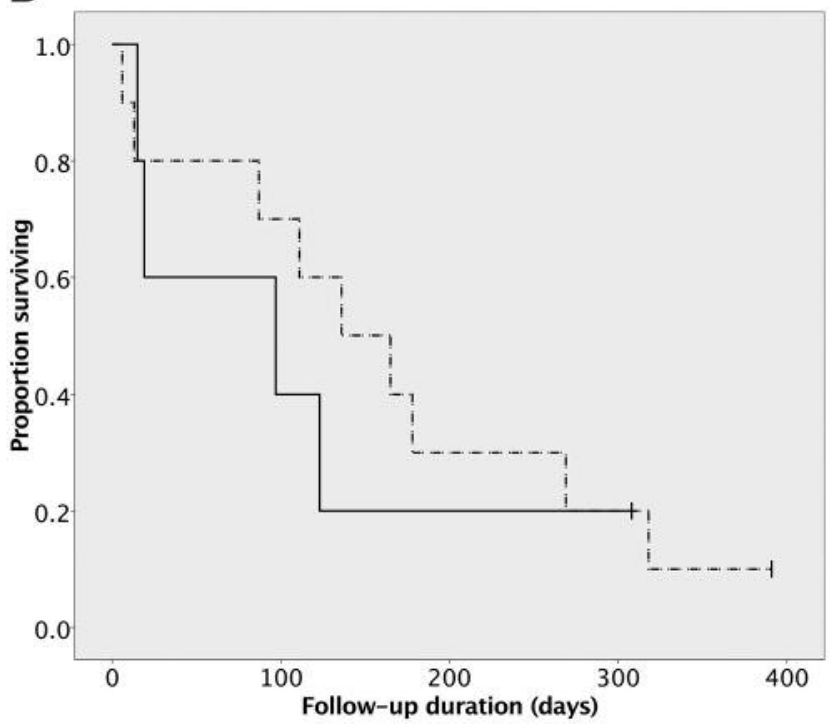

Figure 2. A: Overall survival for patients receiving transarterial chemo-embolization prior to radiotherapy (dashed line) vs. those not (solid line) $(p=0.26)$ B: Overall survival for patients with Child-Pugh score of 8 prior to radiotherapy (dashed line) vs. those with scores of 9-11 (solid line) $(p=0.6)$.

A

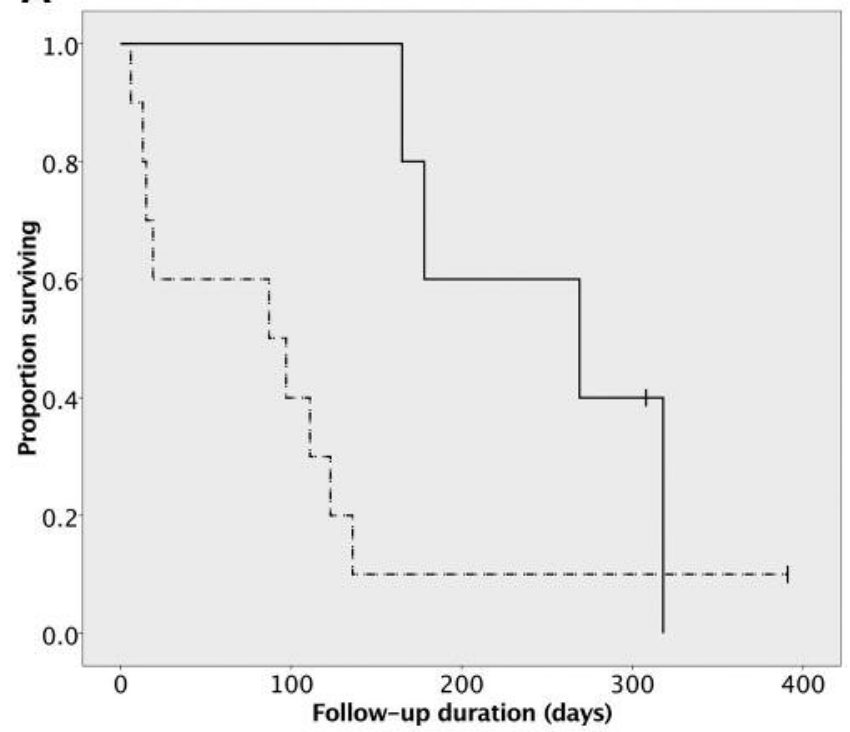

B

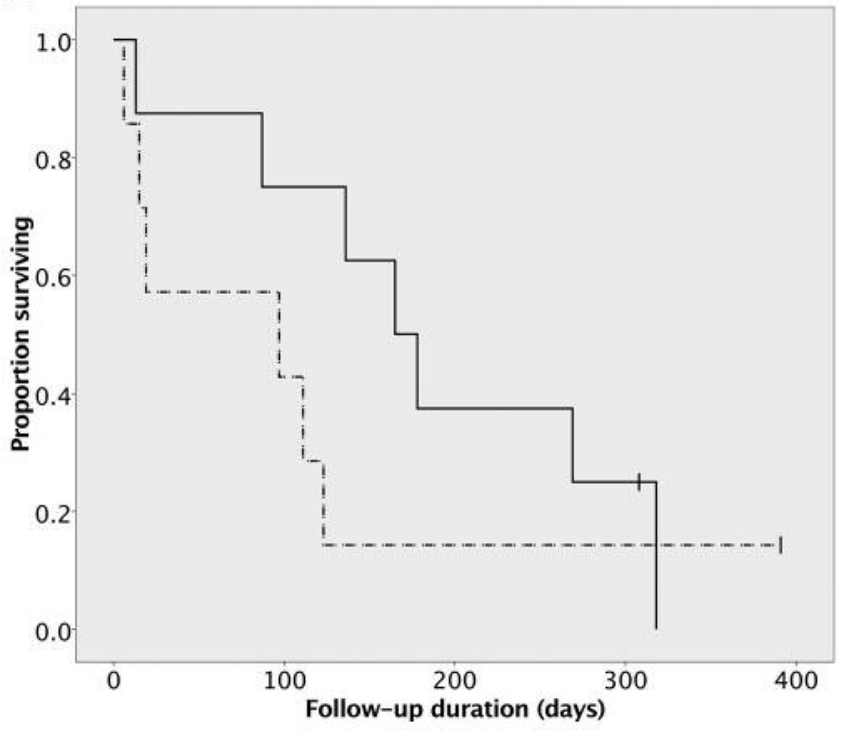

Figure 3. A: Overall survival for patients with any degree of ascites at the beginning of radiotherapy (dashed line) vs. those without ascites (solid line) ( $p=0.056)$. B: Overall survival for patients with any signs or symptoms of decompensated cirrhosis within 30 days of the start of radiotherapy (dashed line) vs. those with clinically stable cirrhosis (solid line) $(p=0.33)$.

SBRT alone. This was despite the fact that in $5 / 6$ of patients treated with TACE in this study hepatic function worsened after TACE (decline in CP score). One explanation for longer survival after TACE is the selection of individuals with indolent tumor biology who survived long enough to be considered for SBRT. Nevertheless, the possibility of combining TACE and SBRT in non-transplant patients may be explored in future safety studies (22). 
We recorded longest survival for the subgroup of five patients who had no ascites at the beginning of SBRT (mean survival of 8.3 months). Conversely, the presence of ascites, particularly severe or medically uncontrolled, appeared to portend a poorer prognosis: a mean survival duration of only 2 months for those with severe/uncontrolled ascites was noted. This observation is in line with the known adverse prognostic significance of ascites for both cirrhosis and HCC $(23,24)$. In addition, ascites complicates radiotherapy planning and delivery (25). Thus, HCC-directed SBRT in the setting of severe cirrhosis has a low probability of success.

We limited this study to patients with severe cirrhosis who were not transplant candidates. Individuals with severe cirrhosis and HCC who are listed for liver transplant represent a different clinical scenario. With impending hepatic transplantation and, therefore, potentially, fewer concerns regarding radiation-induced liver damage, SBRT may play a role as a modality for "bridging to transplant". The goal of SBRT in such cases would be to prevent HCC growth that would place patients outside of the Milan criteria. Additional studies are expected on this subject in the future.

The chief drawbacks of our study are its retrospective design, and therefore it is subject to the usual biases, and a small sample size, leading to low statistical power. However, this report has value due to the dearth of previously published data on the subject, the uniformity of our treatment procedures, and completeness of the follow-up data.

In summary, for transplant-ineligible patients with HCC with cirrhosis of CP score of 8 or more, prognosis after liver SBRT was poor. Patients with tumor persistence or progression after TACE prior to SBRT did not have worse outcomes compared to those treated with SBRT alone for de novo tumors. Patients without any ascites at the time of SBRT had the best prognosis and may be considered for participation in future studies of radiotherapy in this scenario.

\section{References}

1 Colombo M, de Franchis R, Del Ninno E, Sangiovanni A, De Fazio C, Tommasini M, Donato MF, Piva A, Di Carlo V and Dioguardi N: Hepatocellular carcinoma in italian patients with cirrhosis. N Engl J Med 325(10): 675-680, 1991.

2 Sanyal AJ, Yoon SK and Lencioni R: The etiology of hepatocellular carcinoma and consequences for treatment. Oncologist 15(Suppl 4): 14-22, 2010.

3 Jarnagin W, Chapman WC, Curley S, D'angelica M, Rosen C, Dixon E and Nagorney D: Surgical treatment of hepatocellular carcinoma: Expert consensus statement. HPB 12(5): 302-310, 2010.

4 Ioannou GN, Perkins JD and Carithers RL: Liver transplantation for hepatocellular carcinoma: Impact of the meld allocation system and predictors of survival. Gastroenterology 134(5): 1342-1351, 2008.

5 Mazzaferro V, Regalia E, Doci R, Andreola S, Pulvirenti A, Bozzetti F, Montalto F, Ammatuna M, Morabito A and Gennari L: Liver transplantation for the treatment of small hepatocellular carcinomas in patients with cirrhosis. N Engl J Med 334(11): 693-700, 1996.

6 Heimbach JK, Kulik LM, Finn RS, Sirlin CB, Abecassis MM, Roberts LR, Zhu AX, Murad MH and Marrero JA: AASLD guidelines for the treatment of hepatocellular carcinoma. Hepatology 67(1): 358-380, 2018.

7 Parikh ND, Waljee AK and Singal AG: Downstaging hepatocellular carcinoma: A systematic review and pooled analysis. Liver Transpl 21(9): 1142-1152, 2015.

8 Méndez Romero A, Wunderink W, Hussain SM, De Pooter JA, Heijmen BJ, Nowak PC, Nuyttens JJ, Brandwijk RP, Verhoef C and Ijzermans JN: Stereotactic body radiation therapy for primary and metastatic liver tumors: A single institution phase I-II study. Acta Oncol 45(7): 831-837, 2006.

9 Cárdenes HR, Price TR, Perkins SM, Maluccio M, Kwo P, Breen T, Henderson MA, Schefter TE, Tudor K and Deluca J: Phase I feasibility trial of stereotactic body radiation therapy for primary hepatocellular carcinoma. Clin Transl Oncol 12(3): 218-225, 2010.

10 Kirichenko V, Thai NV and Parda DS: Stereotactic body radiation therapy (SBRT) versus radiofrequency ablation (RFA) for unresectable colorectal cancer hepatic metastases: A costeffectiveness analysis. Int J Radiat Oncol Biol Phys 96(2): S163, 2016.

11 Andolino DL, Johnson CS, Maluccio M, Kwo P, Tector AJ, Zook J, Johnstone PA and Cardenes HR: Stereotactic body radiotherapy for primary hepatocellular carcinoma. Int J Radiat Oncol Biol Phys 81(4): 447-453, 2011.

12 Klein $J$ and Dawson LA: Hepatocellular carcinoma radiation therapy: Review of evidence and future opportunities. Int J Radiat Oncol Biol Phys 87(1): 22-32, 2013.

13 Tse RV, Hawkins M, Lockwood G, Kim JJ, Cummings B, Knox J, Sherman M and Dawson LA: Phase I study of individualized stereotactic body radiotherapy for hepatocellular carcinoma and intrahepatic cholangiocarcinoma. J Clin Oncol 26(4): 657-664, 2008.

14 Pan H, Simpson DR, Mell LK, Mundt AJ and Lawson JD: A survey of stereotactic body radiotherapy use in the United States. Cancer 117(19): 4566-4572, 2011.

15 Nair VJ and Pantarotto JR: Treatment of metastatic liver tumors using stereotactic ablative radiotherapy. World J Radiol 6(2): 1825, 2014.

16 Dawson LA, Normolle D, Balter JM, McGinn CJ, Lawrence TS and Ten Haken RK: Analysis of radiation-induced liver disease using the lyman NTCP model. Int J Radiat Oncol Biol Phys 53(4): 810-821, 2002.

17 Dawson LA, Toronto O, Zhu A, Knox J, Krishnan S, Craig T, Guha C, Kachnic L, Gillin MT and Hong TS: Radiation Therapy Oncology Group RTOG 1112 randomized phase III study of sorafenib versus stereotactic body radiation therapy followed by sorafenib in hepatocellular carcinoma. Available at: https://www.rtog.org/Portals/0/RTOG\%20Broadcasts/Attachment s/1112_master_w_update_5.7.13.pdf. (Accessed October 2018)

18 Kirichenko A, Gayou O, Parda D, Kudithipudi V, Tom K, Khan A, Abrams P, Szramowski M, Oliva J and Monga D: Stereotactic body radiotherapy (SBRT) with or without surgery for primary and metastatic liver tumors. HPB 18(1): 88-97, 2016.

19 Urata K, Kawasaki S, Matsunami H, Hashikura Y, Ikegami T, Ishizone S, Momose Y, Komiyama A and Makuuchi M: Calculation of child and adult standard liver volume for liver transplantation. Hepatology 21(5): 1317-1321, 1995. 
20 Kennedy A, Nag S, Salem R, Murthy R, McEwan AJ, Nutting C, Benson A, Espat J, Bilbao JI, Sharma RA, Thomas JP and Coldwell D: Recommendations for radioembolization of hepatic malignancies using yttrium-90 microsphere brachytherapy: A consensus panel report from the Radioembolization Brachytherapy Oncology Consortium. Int J Radiat Oncol Biol Phys 68(1): 13-23, 2007.

21 Culleton S, Jiang H, Haddad CR, Kim J, Brierley J, Brade A, Ringash $\mathrm{J}$ and Dawson LA: Outcomes following definitive stereotactic body radiotherapy for patients with Child-Pugh B or C hepatocellular carcinoma. Outcomes following definitive stereotactic body radiotherapy for patients with Child-Pugh B or C hepatocellular carcinoma. Radiother Oncol 111(3): 412-417, 2014.

22 Combination Chemoembolization and Stereotactic Body Radiation Therapy in Unresectable Hepatocellular Carcinoma. Bethesda (MD): National Library of Medicine (US). Available at: https://clinicaltrials.gov/ct2/show/NCT02513199. (Accessed September 15, 2017)
23 Zipprich A, Garcia-Tsao G, Rogowski S, Fleig WE, Seufferlein $\mathrm{T}$ and Dollinger MM: Prognostic indicators of survival in patients with compensated and decompensated cirrhosis. Liver Int 32(9): 1407-1414, 2012.

24 Hsu C-Y, Lee Y-H, Huang Y-H, Hsia C-Y, Su C-W, Lin H-C, Lee R-C, Chiou Y-Y, Lee F-Y and Huo T-I: Ascites in patients with hepatocellular carcinoma: Prevalence, associated factors, prognostic impact, and staging strategy. Hepatol Int 7(1): 188-198, 2013.

25 Ling TC, Kang JI, Bush DA, Slater JD and Yang GY: Proton therapy for hepatocellular carcinoma. Chin J Cancer Res 24(4): 361-367, 2012.
Received July 17, 2018

Revised October 30, 2018

Accepted November 1, 2018 